\title{
Malnutrition Accelerates Colorectal Cancer Progression through Macrophage Activated by $B$ Cells Immune via Gut Microbiota
}

\section{Xu Chao}

Zhejiang Cancer Hospital

\section{Li Dechuan}

Zhejiang Cancer Hospital

\section{Wang Ziwei}

University of California Davis

\section{Wang Yan}

Ningbo Yinzhou No 2 Hospital

Xu Lu

Zhejiang Chinese Medical University

Chen Haitao

Zhejiang Chinese Medical University

\section{Zhang Bo}

Zhejiang Cancer Hospital

\section{Zhang Runzhe}

Zhejiang Chinese Medical University

Ju Haixing

Zhejiang Cancer Hospital

Yao Qinghua ( $\nabla$ yaoqh@zjcc.org.cn )

Zhejiang Cancer Hospital

\section{Research}

Keywords: Colorectal cancer, Gut microbiota, Malnutrition, Macrophage, B cells, Tumor immune

Posted Date: August 5th, 2020

DOI: https://doi.org/10.21203/rs.3.rs-51243/v1

License: (c) (1) This work is licensed under a Creative Commons Attribution 4.0 International License.

Read Full License 


\section{Abstract}

Background: Malnutrition threatened the clinical outcomes of colorectal cancer (CRC) by reducing patients' responses to anti-cancer treatments and ultimately shortening thesurvival time. Recently malnutrition has been confirmed to play an important role in CRC progress via gut microbiota. However, roles of gut microbiota in the immunopathogenesis of malnutrition and its underlying mechanisms remain inconclusive.

Methods: Patient-Generated Subjective Global Assessment (PG-SGA) was performed to determine the nutrition status in colon cancer patients. 16srRNA sequencing was prepared to explore the dramatic variation of the fecal microbiota among patients with different nutrition status. Fecal microbiota transplantation was used to transplant into $\mathrm{C} 57 \mathrm{BL} / 6 \mathrm{~J}$ mice model and DSS/AOM mice model. Immunohistochemistry and immunofluorescence were applied to test the CD makers. Fluorescenceactivated cell sorting was also prepared to explore the B cells and macrophage from serum and tissues. Enzyme-linked immunosorbent assay and qPCR were used to determine the expression level of cytokines.

Results: In this work, we found the specific microbiota species including Atopobium.vaginae, Selenomonas.sputigena and Faecalibacterium.prausnitzii, which may be considered as the diagnostic biomarkers to help improve poor prognosis in CRC. These microbiota were found to be protumorigenic and adversely affect the nutritional status and overall survival of the animal models. More importantly, our evidence suggesting that these fecal microbiota recruited B cells and macrophage to activate the specific tumor immune in CRC. Depletion of B cells significantly suppressed fecal microbiota inducedM2b polarization, as well as the protumorigenic activity of tumor-associated macrophages in vivo.

Conclusion: gut microbiome in CRC under malnutrition status could upregulate the activity of B cells and protumorigenic macrophage in CRC. Gut microbiome intervention could be a feasible approach to malnutrition-related CRC treatment.

\section{Introduction}

Colorectal cancer (CRC) is one of the most common malignant tumors[1]. However, the pathogenesis of CRC is still not clear. Among all the gut environmental factors, gut microbiota exerts a major role in CRC progression.[2]. In detail, gut microbiota can be implicated in malnutrition, obesity, diabetes, heart disease, cancer, and autoimmune diseases.

Emerging data highlight nutritional status of CRC patients can be impacted by diets due to the fact that gut microenvironment could affect the tumor progression. Malnutrition increases the risks of postoperative complications, anticancer therapy side effects, poor prognosis, and mortality in CRC patients, which leads to increased duration and costs of hospitalization and medical treatments $[3,4]$. Few studies have shown the correlation between nutritional status and gut microbiota in CRC patients. The associated mechanisms involved in how malnutrition-related gut microbiota alteration accelerates CRC progression remain unknown. Additionally, previous studies mainly focused on biomarkers of poor 
prognosis in CRC patients with malnutrition[5]. This work emphasizes the dynamic change of microbiota induced by malnutrition, which provides new insights into diagnostic criteria for malnutritional CRC patients.

The present study aimed to explore the variation of the gut microbiota in CRC patients with different nutritional status. Our results showed that malnutrition altered the CRC patients' gut microbiota, which lead to the dysfunction of the immune system and further predisposing CRC patients to poor prognosis. Moreover, we also demonstrated the role of gut microbiota under the malnutrition status in regulating $\mathrm{B}$ cells and macrophage, which promoted the CRC progression by activating mucosal immunity and further impairing the intestinal barrier. The communications between gut microbiota and malnutrition might directly promote tumor development and progression, resulting in immunoediting of tumors that fosters immune privilege and/or chronic inflammation.

\section{Materials And Methods}

\subsection{Patients Enrollment and Samples Collection}

32 patients (19 male patients and 13 female patients) diagnosed with CRC at Zhejiang Cancer Hospital from 2016-2017, who did not receive preoperative chemotherapy or radiotherapy before surgery, were recruited for this study. Stool samples were collected for $16 \mathrm{~S}$ ribosomal RNA assay. Tumor tissues were flash frozen in liquid nitrogen and kept at $-80^{\circ} \mathrm{C}$ until later use. This study was approved by the ethics committee of Zhejiang Cancer Hospital. Prior to the collection and usage of these clinical materials, every patient offered the informed consent.

\subsection{Patient-Generated Subjective Global Assessment (PG- SGA)}

Patient self-assessment part (including body weight, food intake, symptoms, activity and physical function) and medical staff evaluation part (including nutrition-related disease status, metabolic status, physical examination) composed the PG-SGA score scale. The overall evaluation can be divided into quantitative score and qualitative score. Among them, the results of quantitative evaluation are as follows: The qualitative scores are as follows: (A) good nutrition (0-1 score), (B) suspected / moderate malnutrition ( $2-8$ score), and (C) severe malnutrition ( $\geq 9$ score).

\subsection{Microbial Diversity Analysis}

Microbial DNA was extracted from 32 CRC samples using the QIAamp DNA extraction Kit (Omega Bio-tek, Norcorss, GA, USA) according to manufacturer's protocols. The V3-V5 region of the bacteria $16 \mathrm{~S}$ ribosomal RNA genes were amplified by PCR $\left(95^{\circ} \mathrm{C}\right.$ for $3 \mathrm{~min}$, followed by 30 cycles at $98^{\circ} \mathrm{C}$ for $20 \mathrm{~s}, 58$ ${ }^{\circ} \mathrm{C}$ for $15 \mathrm{~s}$, and $72{ }^{\circ} \mathrm{C}$ for $20 \mathrm{~s}$ and a final extension at $72{ }^{\circ} \mathrm{C}$ for $5 \mathrm{~min}$ ) using primers $341 \mathrm{~F} 5^{\prime}$ - 
CCTACGGGRSGCAGCAG)-3' and 806R 5'-GGACTACVVGGGTATCTAATC-3'. PCR reactions were performed in $30 \mu \mathrm{L}$ mixture containing $15 \mu \mathrm{L}$ of $2 \times$ KAPA Library Amplification Ready Mix, $1 \mu \mathrm{L}$ of each primer (10 $\mu \mathrm{M}), 50 \mathrm{ng}$ of template DNA and ddH2O. After preparation of library, these tags were sequenced on MiSeq/HiSeq platform (Illumina, Inc., CA, USA) for paired end reads of 300/250bp, which were overlapped on their 3 ends for concatenation into original longer tags. DNA extraction, Library construction and sequencing were conducted at Realbio Genomics Institute (Shanghai, China). The results were analyzed by Realbio Genomics Institute (Shanghai, China) and Novogene Genomics Institute (Beijing, China).

\subsection{Fecal Microbiota Transplantation (FMT)}

Stools from patients, who were evaluated as moderate malnutrition or severe malnutrition by PG-SGA and had not received surgery, were freshly collected on the day of transplant. Donor stool was weighed and diluted with $1 \mathrm{ml}$ of saline per $0.1 \mathrm{~g}$ of stool. Briefly, the stool was steeped in saline for about $15 \mathrm{~min}$, shaken and then centrifuged at $800 \mathrm{rpm}$ for $3 \mathrm{~min}$. The supernatant was obtained for intragastric administration ( $0.3 \mathrm{~mL}$ per mouse). The transplantation was performed within $4 \mathrm{~h}$ upon stool collection.

\subsection{Immunohistochemistry (IHC) and Immunofluorescence}

Formalin-fixed and paraffin-embedded mice colon samples were cut into $4 \mu \mathrm{m}$ sections and incubated in an oven overnight. After the sections were deparaffinized, EDTA ( $\mathrm{pH}$ 9.0) was prepared for antigen retrieval in a microwave for $20 \mathrm{~min}$. Sections were incubated with antibodies against mouse Ki-67 (Abcam) and then stained in Envision System (Dako). Immunohistochemical results were tested by two independent pathologists who were blinded to the clinical assessments of patients. Images were obtained by a KFBio microscope with K-ViEWER software. For immunofluorescence analysis, frozen sections were stained with mouse anti-B220 (Abcam) plus rabbit anti-F4/80 (Abcam), followed by FITCconjugated anti-mouse IgG plus TRICT-anti rabbit IgG (Affinity). DAPI (Beyotime) was prepared to stain the cell nucleus. Positive cells were detected by confocal microscopy.

\subsection{Quantitative Polymerase Chain Reaction (qPCR)}

mRNA was extracted from tissues with TRIzol reagent (Invitrogen, Carlsbad, CA, USA) according to the manufacturer's protocol. SYBR Green Master Mix (Invitrogen, Carlsbad, CA, USA) kit was prepared to determine the mRNA expression by qPCR. Detailed information about all primers used were listed in Appendix Table S1.

\subsection{Fluorescence-Activated Cell Sorting (FACS)}

The detailed protocol of flow cytometry has been described in the previous article[6]. 


\subsection{Animal Model and in vivo Suppression of B Cells or Inhibition of Monocyte/Macrophage Activities}

Eight-week-old male and female C57BL/6J mice were purchased from SLAC (Shanghai, China). Mice were randomly divided into different groups, housed in a specific pathogen-free level of the animal facility at laboratory animal center of Zhejiang Chinese Medical University under standard conditions. All procedures and animal handlings were performed in the agreement with the ethical guidelines for animal studies. Animal experiments were approved by the Institutional Animal Care and Use Committee of Zhejiang Chinese Medical University and Zhejiang Cancer Hospital. Construction of DSS/AOM mice model referred to Bobak Parang's article and the detail process was shown in Fig.7A. In some cases, the mice were first treated with an anti-CD20 antibody $(10 \mathrm{mg} / \mathrm{kg}$ body weight) for 6 days. Thereafter, they were injected with $\mathrm{GdCl} 3(10 \mathrm{mg} / \mathrm{kg}$ body weight) plus anti-CD20 antibody (10 mg/kg body weight) every 3 days. After 21 days, tumors were harvested for subsequent immunohistochemical staining of $F 4 / 80$ (Abcam) and B220 (Abcam). The expressions of M2b markers (IL-10 and CCL1) and M1 marker IL-12 in tissues was analyzed by qPCR.

\subsection{Enzyme-Linked Immunosorbent Assay (ELISA)}

Concentrations of IL-10, IL-12, and CCL1 in mice tissue and blood were detected using ELISA kits according to the manufacturers' instructions (eBioscience for IL-10, IL-12 and CCL1).

\subsection{Statistical Analysis}

All the experimental data were analyzed by GraphPad Prism 8.0 and results were shown as mean \pm standard deviation (SD). Comparisons between two groups were performed by Student's t-test. Multiple comparisons were analyzed by one-way ANOVA. Survival analysis was analyzed using the Kaplan-Meier method. $\mathrm{P}<0.05$ was considered statistically significant.

\section{Results}

\subsection{The Characteristics of Participants}

$32 \mathrm{CRC}$ patients were enrolled in this study. Among them, six were assessed as $0-1$ score (considered as PG-SGA 1), eleven as 2-3 score (considered as PG-SGA 2), fourteen as 4-8 score (considered as PG-SGA 3 ), and one as $\geq 9$ score (considered as PG-SGA 4). Furthermore, according to the PG-SGA, fifteen of them needed nutritional intervention. 11 patients were diagnosed with colon cancer and the other 21 patients with rectal cancer. Primary tumor sizes were significantly different among triages based on PGSGA score. The biochemical examination of blood in the all these patients found that the mean level of albumin (ALB) was $38.33 \pm 5.58 \mathrm{~g} / \mathrm{L}$, of prealbumin (PAB) was $210.81 \pm 72.07 \mathrm{~g} / \mathrm{L}$, of transferrin (TF) was 
$2.43 \pm 0.54 \mathrm{~g} / \mathrm{L}$. Accordingly, the BMI index was $23.23 \pm 3.33$. Those parameters of different PG-SGA triages are shown in Table 1. There was no difference found in BMI, blood ALB and PAB levels among all the patients. Notably, PG-SGA 4 had the lowest levels of both blood ALB and PAB. TF level in PG-SGA 4 was significantly lower than all the other groups.

Table 1

Clinical characteristics of the study subjects in PGSGA screening assessment

\begin{tabular}{|llllll|}
\hline items & PG-SGA1 & PG-SGA2 & PG-SGA3 & PG-SGA4 & P value \\
\hline $\begin{array}{l}\text { Gender } \\
\text { (male vs female) }\end{array}$ & $(2 / 4)$ & $(6 / 5)$ & $(6 / 7)$ & $(1 / 1)$ & 0.87 \\
\hline $\begin{array}{l}\text { Primary tumor size } \\
\text { (colon vs rectum) }\end{array}$ & $(1 / 5)$ & $(1 / 10)$ & $(7 / 6)$ & $(2 / 0)$ & 0.019 \\
\hline BMI & $25.05 \pm 4.70$ & $23.38 \pm 3.00$ & $22.37 \pm 3.06$ & $22.51 \pm 0.41$ & 0.44 \\
ALB & $40.62 \pm 3.26$ & $38.95 \pm 4.01$ & $37.4 \pm 7.46$ & $34.05 \pm 1.06$ & 0.057 \\
\hline PAB & $219.33 \pm 47.51$ & $242.18 \pm 51.56$ & $195.31 \pm 85.79$ & $113.5 \pm 38.89$ & 0.085 \\
\hline TF & $3.41 \pm 0.47$ & $2.27 \pm 0.34$ & $2.38 \pm 0.51$ & $1.45 \pm 0.28^{*}$ & 0.0001 \\
\hline
\end{tabular}

Table 1, Clinical characteristics of the study subjects in PG-SGA screening assessment. BMI, Body Mass Index. ALB, Albumin. PA, Prealbumin. TF, Transferrin. ( $\left.{ }^{*}, \mathrm{P}<0.05\right)$.

\subsection{Fecal Microbiota Analysis in CRC Patients with Malnutrition}

\section{Community richness and diversity}

To determine the relationship between the intestinal bacterial pattern and nutrition status, we used $16 \mathrm{~S}$ rRNA sequencing to analyse the enteric bacterial profile in patients feces. The number of different species for each PG-SGA trigage was generated by Venn Graph and shown in Fig. 1A. The ACE, Chao1, Simpson and Shannon indices were different among the four groups. The community richness in PG-SGA 3 and PG-SGA 4 are lower than that in PG-SGA 1 and PG-SGA 2 individuals. (Fig. 1B-E).

\section{Significant difference in the microbiota between individuls with malnutrition assessed by PG-SGA}

To determine if there are differences in the microbiota among PG-SGA groups, samples from all the patients were analyzed for bacterial communities. The overall microbial composition of heat map in genus level is shown in Fig. 2A upper as well as top 15 heat map in genus level of each PG-SGA group shown in Fig. 2A lower. According to heatmap result, there are significant differences in the four PG-SGA 
groups. Meanwhile, We also conducted a principal coordinates analysis based on the Upgma Bray-Curtis dissimilarity distance to explore the different phylum in PG-SGA groups. The phylum level of Proteobacteria and Firmicutes indicated they were the the key microbiota species in these individuals. Consistently, PG-SGA 4 had lower level of bacteroidetes, Verrucumicrobia, Fusobacteria, Actinobacteria, Tenericutes, Cyanobacteria, Synergistetes, and Acidobacteria. Fusobacteria and Verrucumicrobia were found to be more predominant in PG-SGA 3 than in other three groups. However PG-SGA 2 showed the same changes except for bacteroidetes (Fig. 2B), which directly reflected the change of microbiota in genus level. We further analyzed the enterotype of the gut microbiota, which is another general measurement of the gut microbiota composition. By using the Principal Co-ordinates Analysis (Fig. 2C) and Principal Component Analysis (Fig. 2D) scores based on the relative abundance of the fecal microbiota at genera levels, we revealed a significant separation in the bacterial community composition between four group individuals. Based on the enterotype results, the cohort was divided into two clusters, namely enterotype 1 and enterotype 2, each of which accounted for different proportions in PG-SGA groups (Fig. 2E).

\section{Correlation between gut microbiota and bioclinical variables}

We further investigated potential associations between previously identified key microbial genera and malnutrition index (Fig. 3). According to RDA/CCA analysis, blood ALB and PA levels are correlated with gut microbiota in these malnutrition individuals (Fig. 3A and 3B). The Enterocoocaceae group had a close relationship with many clinical parameters, where it showed negative correlations with ALB $(P<0.05)$ and PA blood levels $(P<0.05)$. Additionally, blood level of PA had significant correlations $(P<0.05)$ with Gemellaceae, Enterocoocaceae, lactobacillaceae, and Peptostreplococcaceae. ALB blood level was also negatively correlated with Erysipelotrichaceae $(P<0.05)$.

\section{Prediction of malnutrition metabolites via gut microbiota}

KEGG pathway was analyzed by LDA Effect Size to further predict potential associations between key microbial genera and metabolic disorders in malnutrion patients (Fig. 4A). PG-SGA 4 group showed less activity of metabolic pathways such as "oxygenic photoautotrophy", "cyanobacteria", "photoautotrophy", and "phototrophy" pathways than other groups. Levels of "animal parasites and symbionts", "chemoheterotrophy", "fermentation", "mammal gut" were also lower in PG-SGA 4 group than in other groups. On the other hand, higher levels of "human pathogens" and "aromatic compound degradation" were found in PG-SGA4 than in other groups. It was discovered that "Metabolism" and "Neurodegenerative diseases" were the main changes characterized in PG-SGA 3 and PG-SGA 4, in comparison to the discovery that "Environmental adaptation" was the main one in PG-SGA 1 and PG-SGA 2 by KEGG pathway. Moreover, specific changes in PG-SGA 1 and PG-SGA 2 groups were mainly aggregated in "Plant pathogen interaction", "Cysteine and methionine metabolism", "Basal transcription factor", "Thiamine metabolism", "Cytoskeleton proteins", "Ribosome biogenesis", and "Lysine biosynthesis". On the other hand, PG-SGA 3 and PG-SGA 4 groups had more changes on the 
"Glycosyltransferases", "Protein folding and associated processing" and "Metabolism of cofactors and vitamins" (Fig. 4B).

\section{Establishment of disease surveillance model in malnutrition}

Receptor operating characteristic (ROC) curve were prepared to examine the diagnostic ability of classification and prediction of different microbiota species (Fig. 5A-B). We selected the three bacterium high in genera level (Atopobium, Selenomonas and Faecalibacterium), which could be potentially evaluated to predict and classify the malnutrition for PG-SGA 3 and PG-SGA 4 groups. Based on ROC result, we evaluated the capacity of these three bacterium in predicting the prognosis in CRC. Among the three species, Atopobium.vaginae (A.vaginae) was the most capable one to distinguish the two groups (PG-SGA1 and PG-SGA2 vs PG-SGA3 and PG-SGA4, P<0.01). Thus, we conclude that $A$.vaginae could exert as a biomarker in overall survival in CRC, but Selenomonas. sputigena (S. sputigena) and Faecalibacterium. prausnitzii (F. prausnitzii) failed.

\subsection{Gut Microbiota in Malnutrition Promotes CRC Progression}

\section{Gut microbiota in malnutrition is considered as a co- carcinogenic factor}

To verify the biological effects of the gut microbiota from CRC patients with malnutrition, we used FMT to colonize the gut microbiota of C57BL/6J mice by gavage. Interestingly, mice treated with PG-SGA 3 and PG-SGA 4 fecal samples molted, which was not observed in the control group gavaged by saline. Notably, feces from PG-SGA 4 group may lead to a worse molting by triggering a stronger autoimmune response (Fig. 6A). 16S rRNA sequencing confirmed mice gavaged with PG-SGA 4 fecal samples were colonized by Atopobium in the intestine (Fig. 6B). Consistently, higher grade of intraepithelial neoplasia was observed in mice transplanted with PG-SGA 4 fecal samples compared to those transplanted with PG-SGA 3 feces (Fig. 6C). We next used IHC assay to test the cell proliferation by Ki-67 marker. The expression of Ki-67 was increased in both malnutrition groups, indicating the cell proliferation was increased in both malnutrition group.. Mice transplanted with fecal samples from malnutrition CRC patients showed poorer results in the nutritional indicators such as TF, ALB, PA and body weight, which may indicate the role of gut microbiota played in malnutrition development (Fig. 6D), which ultimately lead to poor prognosis (Fig. 6E). Additionally, PG-SGA 4 group expressed higher level of Ki-67 than PG-SGA3 group (Fig. 6F). To investigate the mechanism, we examined the immune effect in groups treated with FMT. We observed a remarkable accumulation of $B$ cells, but not $T$ cells, in the serum using FACS. Meanwhile, B cells accumulation recruited more macrophages rather than monocytes in the treated groups. To verify these results, we used immunofluorescence to explore B cells and macrophage in the intestinal tissues from treated groups. The results were consistent with the serum outcomes (Fig. 6G). We subsequently used ELISA and qPCR to analyze the cytokines secreted by macrophages in the serum and intestine tissues. 
The treated groups expressed higher levels of M2b markers IL-10 and CCL1, as well as M1 marker IL-12, than those in control group (Fig. $6 \mathrm{H}$ ). These results may indicate the feces from malnutrition patients would trigger the intestinal mucosal immune system via recruitment of B cells to activate macrophage.

\section{Gut microbiota from malnutrition accelerates CRC progression in vivo}

The above results suggested microbiota could be considered as the co-carcinogenic factor in CRC progression in patients under malnutrition status. Thus, we aimed to validate the role of microbiota in the DSS/AOM mice model. Consistent with regular C57BL/6J/6J mice model, the DSS/AOM mice models transplanted with feces from malnutrition patients groups had stronger autoimmune response accompanied with the increased numbers of intestinal adenoma comparing to the control group (Fig. 7B). The levels of nutritional indicators also decreased significantly in PG-SGA 3 and PG-SGA 4 group (Fig. 7C). Accordingly, we found both $B$ cells and macrophages were largely infiltrated in the intestine in FMT treated groups, especially in PG-SGA 4 group, by FACS (Fig. 7D). PG-SGA 4 group exhibited a stronger autoimmune response, higher humbers of intestinal adenoma, poorer nutritional status and greater ability to promote the proliferation of adenoma (Fig. 7E) than the other two groups. The expression level of M2b markers IL-10 and CCL1 in PG-SGA 4 group was higher than other groups. Surprisingly, the accumulation of B cells and macrophages in serum did not change significantly between groups (Fig. 7F). In the meanwhile, the expression of M1 marker IL-12 was found to be highest in control group (Fig. 7G).

\section{Suppression of B cells and/or inhibition of monocyte/macrophage activities attenuates the CRC progression induced by microbiota from malnutritional patients}

Our next goal was to determine whether blocking B cells or macrophage in DSS/AOM mice models could attenuate the CRC progression mediated by gut microbiota from PG-SGA 4 malnutritional patients. With the injection of anti-CD20 antibody, $\mathrm{GdCl}_{3}$, or anti-CD20 plus $\mathrm{GdCl}_{3}$ respectively, depletion of $\mathrm{B}$ cells and suppression of macrophage activities were significant in intestinal tissue (Fig. 8A). The tumor volume and Ki-67 expression implied that anti-CD20 treatment had greater anticancer effects than anti-CD20 plus $\mathrm{GdCl}_{3}$ treatment in DSS/AOM mice model. Nevertheless, inhibition of monocyte/macrophage activities only may not suppress tumor progression effectively (Fig. 8B, 8C), stressing a key role of B cells in the $\mathrm{CRC}$ progression promoted by malnutrition-induced gut microbiota alteration.

\section{Discussion}

Substantial evidence have shown that malnutrition acts as an independent prognostic factor in CRC. It increased the risk of tumor progression acceleration by reducing patient's response to anticancer 
treatment, which ultimately lowers the survival rate [4]. Malnourished patients with CRC including those treated with surgery, radiotherapy and/or chemotherapy, are at higher risks of suffering from postoperative complications, prolonged length of hospitalization causing heavy economic burden on family and society, a poorer quality of life, and an increased mortality [7-9]. The present study demonstrated that gut microbiota could be an important factor to recruit $B$ cells and macrophages, which further activate mucosal immunity to accelerate the progression in CRC patients with malnutrition.

Recently, a number of metagenomics studies have illustrated associations between the altered gut microbiota and malnutrition[10,11]. However, these studies examined neither metabolic or immune functions, nor interactions between individual species of gut microbiota during in malnutrition subjects. We discovered the microbiota composition exhibited significant differences under malnutrition status in CRC. We next identified Atopobium, Selenomonas and Faecalibacterium may predict the different malnutrition status in $\mathrm{CRC}$ patients using ROC analysis. It brought to our attention that Atopobium and Fusobacterium were identified as sarcoidosis-associated bacteria, which may enable new insights into the pathogenesis and treatment of CRC[12]. In CRC, the abundance of Atopobium parvulum and Actinomyces odontolyticus, were significantly increased only in multiple polypoid adenomas and/or intramucosal carcinomas, indicating those two bacteria may be considered as the microbiome therapy target in CRC[13]. These studies provided evidence that not only the single effect, but also synergistic effects of Atopobium with other gut microbiota such as Fusobacterium, Stomatobaculum,and Actinomyces may lead to different clinical outcomes. In this regard, our results showed A.vaginae correlated with $S$. sputigena and F. prausnitzii could provide support to establish new therapeutic strategies for CRC patients with malnutrition.

Growing attention has been received on the link between the microbiome and the development of CRC with malnutrition [14]. Our research aimed to explore the potential of utilizing microbiota as malnutritional biomarkers, and the possibility of modifying the microbiota as a prevention or treatment for CRC with malnutrition. For instance, Selenomonas, which was positively correlated with BDCA2+ pDCs and Foxp3 + Tregs[15] and recognized as a tongue coatings biomarker in patients with gastric cancer[16], has been identified to be more abundant in right colon cancer (RCC) patients than left colon cancer (LCC) patients[17]. According to Ru et al, the microbiota panel including Selenomonas, Clostridium, Bacteroidetes Slackia, Burkholderiales and Veillonellaceae were closely related to CRC patients with hyperlipoidaemia[18]. Similarly, by combining the nutritional assessment tool, our clinical serum outcomes and gut microbiome data suggested Enterocoocaceae had negative correlations with ALB and PA levels. Additionally, PA level showed negative correlations with Gemellaceae, Enterocoocaceae, lactobacillaceae and Peptostreplococcaceae. ALB level also negatively correlated with Erysipelotrichaceae. Moreover, Selenomonas combined with Atopobium and Faecalibacterium would be a fantastic panel for diagnosis or treatment purposes in CRC with malnutrition. We further verified how gut microbiota regulated the CRC progression in malnutrition. Importantly, we found that a FMT with malnutrition fecal led to an accelerated growth of colonic adenoma in C57BL/6J/6J mice model and DSS/AOM mice model established with C57BL/6J/6J mice. FMT experiments also confirmed the models 
exerted poor outcomes in nutritional indicators such as TF, ALB, PAB, and body weigh. Overall, gut microbiota alteration induced by malnutrition could lead to the carcinogenesis in CRC.

In regards to mechanisms, it was characterized that microbiota may activate the mucosal immune response by triggering $B$ cell infiltration in the intestine, recruiting macrophages to promote the CRC progression. B cel infiltration, in particular, plasma cell infiltration, has a significant impact on tumour progression and prognosis in CRC [19]. Meanwhile, tumor macroenvironment could affect the differentiation, function and distribution of B lymphocytes, switching B cells to pro-, rather than anti-, tumoral phenotype[20]. B cells could aggravate the inflammatory response and promote the colitis to colorectal cancer[21, 22]. This raise the question - did the malnutrition affect the B cells infiltration in CRC or did B cells worsen the malnutrition status? B cells have been demonstrated as an essential factor associated with malnutrition[23, 24]. Previous research showed under anorexia nervosa, increased number of $B$ lymphocyte associated with body mass loss drives the relative lymphocytosis, leading to the poor outcome[25]. Many studies have discovered the prognostic role of B cells in CRC on malnutrition status, yet how the $B$ cells regulated the malnutrition still remain unknown. We noticed both $B$ cells and macrophages were regulated by these microbiota, and B cells, particularly plasma cells, could lead macrophages to polarization and differentiate into M1 like or M2 like macrophages, which present proinflammatory or pro-tumor effects via cytokine IL-17 or IL-10 secretion $[6,26]$. The in vivo results of C57BL/6J/6J model showed higher levels of M2b markers (IL-10 and CCL1) and M1 marker IL-12 in serum and intestinal tissue after FMT with malnutrition feces. DSS/AOM mice model also exerted the higher level of IL-10 and CCL1, which may indicate gut microbiota malnutrition would promote the progression of chronic colitis to tumor via macrophage polarization by $B$ cells. The above results urge our team to explore deeper the mechanisms of $B$ cells in regulation of macrophages via the microbiota under malnutrition status in CRC in the future. Elucidating these mechanisms may lead to the development of new modulation practices for malnutrition-related CRC prevention and treatment by targeting the gut microbiota. This potential therapeutic strategy may improve the poor prognosis of CRC patients with malnutrition.

Our research provides important insights into role of gut micrbiota under malnutrition status in CRC patients in regulation of intestinal immune. These findings give a novel understanding of association between gut microbiota and malnutrtion, illuminating a new microbiome-based methodology for diagnosing and curing CRC in malnutriton. Given the lack of knowledge on the underlying mechanisms, further studies are essential to understand how the gut microbiota under malnutrition status affects $B$ cells immune function in CRC.

\section{Abbreviations}

ALB, Albumin

$\mathrm{CRC}$, Colorectal caner

ELISA, Enzyme-linked immunosorbent assay 
FACS, Fluorescence-activated cell sorting

FMT, Fecal microbiota transplantation

IHC, Immunohistochemistry

IL, Interleukin

KEGG, Kyoto encyclopedia of genes and genomes

LCC, Left colon cancer

PCR, Polymerase chain reaction

PG-SGA, Patient-generated subjective global assessment

PA, Prealbumin

ROC, Receiver operating characteristic curve

RCC, Right colon cancer

SD, Standard deviation

TF, Transferrin

\section{Declarations}

\section{Funding Information}

This work was supported by the National Natural Science Foundation of China (81873121), the key project of Chinese Medical Science and Technology Foundation of Zhejiang Province (2017ZZO04, 2019ZZ003), the key project of Natural Science Foundation of Zhejiang Province (Z2OH290002), and the normal project of Medical Science and Technology Foundation of Zhejiang Province (2020KY070).

\section{Acknowledgements}

We thank Dr. Ding Xilai from Westlake Institute for Advanced Study for technical help with FACS, Dr. Chen Cheng from Zhejiang Chinese Medical University for technical help with immunofluore, Prof. Chen Guoping from Zhejiang Cancer Hospital for technical help with IHC.

\section{Author contributions}


Conceived and designed the experiments: XU Chao and YAO Qinghua. Prepared the manuscript: XU Chao and WANG Ziwei. Performed the experiments: XU Chao, LI Dechuan, XU Lu, ZHANG Runzhe, and JU Haixing. Analyzed the data: WANG Ziwei, CHEN Haitao, and WANG Yan. Funding owner: YAO Qinghua and XU Chao

\section{Conflicts}

The author(s) declare that they have no conflict of interest.

\section{References}

1. Miller KD, Siegel RL, Lin CC, Mariotto AB, Kramer JL, Rowland JH, Stein KD, Alteri R, Jemal A. Cancer treatment and survivorship statistics, 2016. CA Cancer J Clin. 2016;66:271-89.

2. Garrett WS. The gut microbiota and colon cancer. Science. 2019;364:1133-5.

3. Almasaudi AS, McSorley ST, Dolan RD, Edwards CA, McMillan DC. The relation between Malnutrition Universal Screening Tool (MUST), computed tomography-derived body composition, systemic inflammation, and clinical outcomes in patients undergoing surgery for colorectal cancer. Am J Clin Nutr. 2019.

4. Gallois C, Artru P, Lièvre A, Auclin E, Lecomte T, Locher C, Marthey L, Zaimi Y, Faroux R, Pernot S, et al. Evaluation of two nutritional scores' association with systemic treatment toxicity and survival in metastatic colorectal cancer: an AGEO prospective multicentre study. Eur J Cancer. 2019;119:35-43.

5. Hughes RL, Kable ME, Marco M, Keim NL. The Role of the Gut Microbiome in Predicting Response to Diet and the Development of Precision Nutrition Models. Part II: Results. Adv Nutr. 2019.

6. Liu RX, Wei Y, Zeng QH, Chan KW, Xiao X, Zhao XY, Chen MM, Ouyang FZ, Chen DP, Zheng L, et al. Chemokine (C-X-C motif) receptor 3-positive B cells link interleukin-17 inflammation to protumorigenic macrophage polarization in human hepatocellular carcinoma. Hepatology. 2015;62:1779-90.

7. Hu WH, Eisenstein S, Parry L, Ramamoorthy S. Preoperative malnutrition with mild hypoalbuminemia associated with postoperative mortality and morbidity of colorectal cancer: a propensity score matching study. Nutr J. 2019;18:33.

8. Cavagnari M, Silva TD, Pereira M, Sauer LJ, Shigueoka D, Saad SS, Barão K, Ribeiro C, Forones NM. Impact of genetic mutations and nutritional status on the survival of patients with colorectal cancer. BMC Cancer. 2019;19:644.

9. Oh SY, Koh SJ, Baek JY, Kwon KA, Jeung HC, Lee KH, Won YW, Lee HJ. Validity and Reliability of Korean Version of Simplified Nutritional Appetite Questionnaire in Patients with Advanced Cancer: A Multicenter, Longitudinal Study. Cancer Res Treat. 2019;51:1612-9.

10. Ticinesi A, Lauretani F, Milani C, Nouvenne A, Tana C, Del Rio D, Maggio M, Ventura M, Meschi T. Aging Gut Microbiota at the Cross-Road between Nutrition, Physical Frailty, and Sarcopenia: Is There 
a Gut-Muscle Axis. Nutrients. 2017;9.

11. Milani C, Duranti S, Bottacini F, Casey E, Turroni F, Mahony J, Belzer C, Delgado Palacio S, Arboleya Montes S, Mancabelli L, et al. The First Microbial Colonizers of the Human Gut: Composition, Activities, and Health Implications of the Infant Gut Microbiota. Microbiol Mol Biol Rev. 2017;81.

12. Zimmermann A, Knecht H, Häsler R, Zissel G, Gaede Kl, Hofmann S, Nebel A, Müller-Quernheim J, Schreiber S, Fischer A. Atopobium and Fusobacterium as novel candidates for sarcoidosisassociated microbiota. Eur Respir J. 2017;50.

13. Scott AJ, Alexander JL, Merrifield CA, Cunningham D, Jobin C, Brown R, Alverdy J, O'Keefe SJ, Gaskins HR, Teare J, et al. International Cancer Microbiome Consortium consensus statement on the role of the human microbiome in carcinogenesis. Gut. 2019;68:1624-32.

14. Yachida S, Mizutani S, Shiroma H, Shiba S, Nakajima T, Sakamoto T, Watanabe H, Masuda K, Nishimoto $\mathrm{Y}$, Kubo M, et al. Metagenomic and metabolomic analyses reveal distinct stage-specific phenotypes of the gut microbiota in colorectal cancer. Nat Med. 2019;25:968-76.

15. Ling Z, Shao L, Liu X, Cheng Y, Yan C, Mei Y, Ji F, Liu X. Regulatory T Cells and Plasmacytoid Dendritic Cells Within the Tumor Microenvironment in Gastric Cancer Are Correlated With Gastric Microbiota Dysbiosis: A Preliminary Study. Front Immunol. 2019;10:533.

16. Xu J, Xiang C, Zhang C, Xu B, Wu J, Wang R, Yang Y, Shi L, Zhang J, Zhan Z. Microbial biomarkers of common tongue coatings in patients with gastric cancer. Microb Pathog. 2019;127:97-105.

17. Kim K, Castro E, Shim H, Advincula J, Kim YW. Differences Regarding the Molecular Features and Gut Microbiota Between Right and Left Colon Cancer. Ann Coloproctol. 2018;34:280-5.

18. Han S, Pan Y, Yang X, Da M, Wei Q, Gao Y, Qi Q, Ru L. Intestinal microorganisms involved in colorectal cancer complicated with dyslipidosis. Cancer Biol Ther. 2019;20:81-9.

19. Berntsson J, Nodin B, Eberhard J, Micke P, Jirström K. Prognostic impact of tumour-infiltrating B cells and plasma cells in colorectal cancer. Int J Cancer. 2016;139:1129-39.

20. Mion F, Vetrano S, Tonon S, Valeri V, Piontini A, Burocchi A, Petti L, Frossi B, Gulino A, Tripodo C, et al. Reciprocal influence of $B$ cells and tumor macro and microenvironments in the ApcMin/+ model of colorectal cancer. Oncoimmunology. 2017;6:e1336593.

21. De Arcangelis $A$, Hamade $H$, Alpy F, Normand $S$, Bruyère $E$, Lefebvre $O$, Méchine-Neuville A, Siebert $S$, Pfister V, Lepage $\mathrm{P}$, et al. Hemidesmosome integrity protects the colon against colitis and colorectal cancer. Gut. 2017;66:1748-60.

22. Lin S, Haque A, Raeman R, Guo L, He P, Denning TL, El-Rayes B, Moolenaar WH, Yun CC. Autotaxin determines colitis severity in mice and is secreted by B cells in the colon. FASEB J. 2019;33:3623-35.

23. González-Gutiérrez AM, Ortiz-Muñiz R, García-Rodríguez M, Cortés-Barberena E. Phosphorylated ATM and $H 2 A X$ in $T$ and $B$ lymphocytes from rats with moderate and severe malnutrition. DNA Repair (Amst). 2019;83:102640.

24. Floreani A, Restrepo-Jiménez P, Secchi MF, De Martin S, Leung P, Krawitt E, Bowlus CL, Gershwin ME, Anaya JM. Etiopathogenesis of autoimmune hepatitis. J Autoimmun. 2018;95:133-43. 
25. Elegido A, Graell M, Andrés P, Gheorghe A, Marcos A, Nova E. Increased naive CD4+ and B lymphocyte subsets are associated with body mass loss and drive relative lymphocytosis in anorexia nervosa patients. Nutr Res. 2017;39:43-50.

26. Wei Y, Lao XM, Xiao X, Wang XY, Wu ZJ, Zeng QH, Wu CY, Wu RQ, Chen ZX, Zheng L, et al. Plasma Cell Polarization to the Immunoglobulin G Phenotype in Hepatocellular Carcinomas Involves Epigenetic Alterations and Promotes Hepatoma Progression in Mice. Gastroenterology. 2019;156:1890-904.e16.

\section{Figures}

\section{Figure 1}
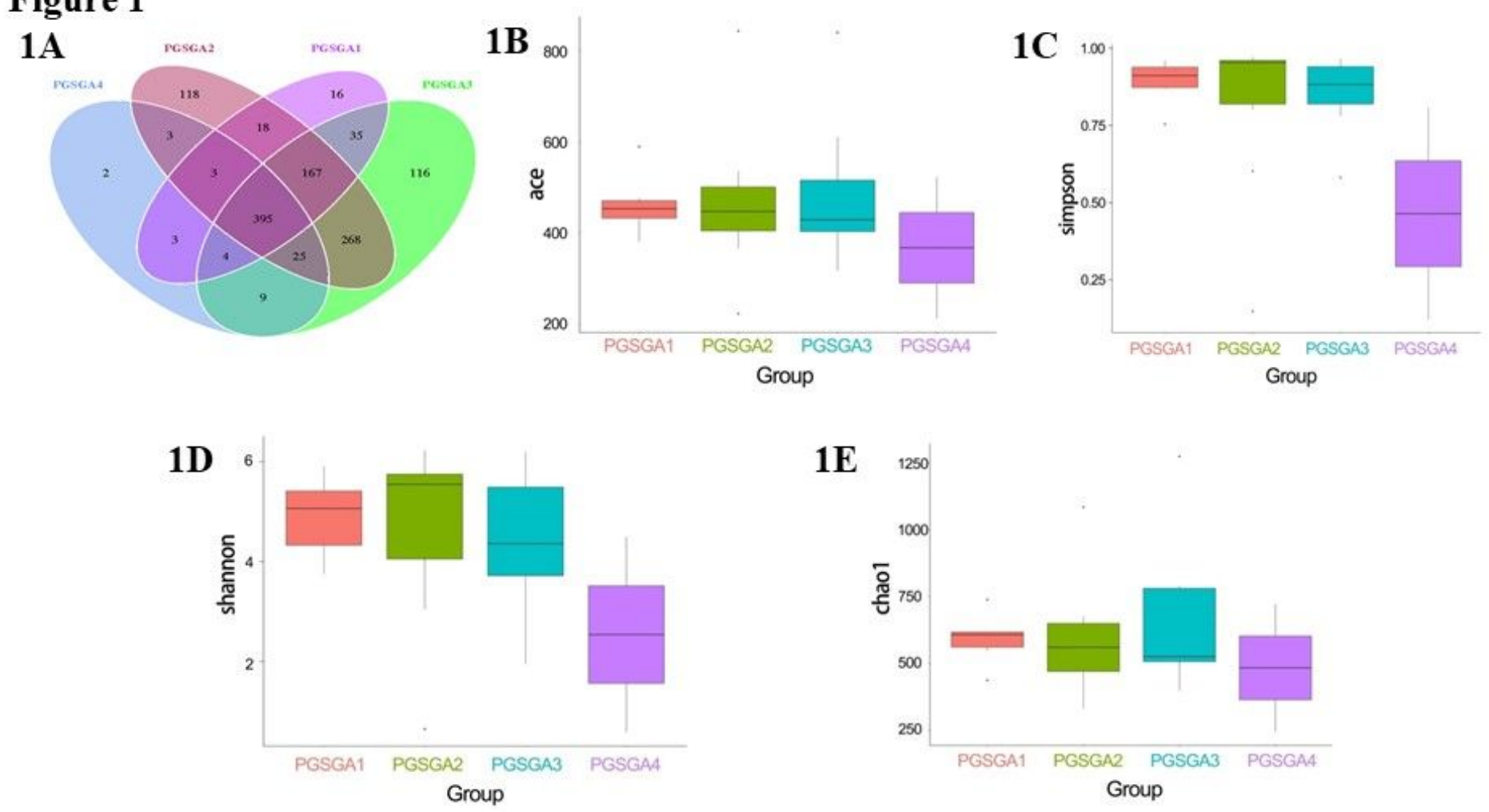

\section{Figure 1}

Diversity analysis of gut microbiota from the different grade of PG-SGA level in CRC patients. A:Venn Graph of the four grade PG-SGA groups. B-E:The ACE (B), Simpson (C), Shannon (D), and Chao1 (E) indices among in PG-SGA groups. 


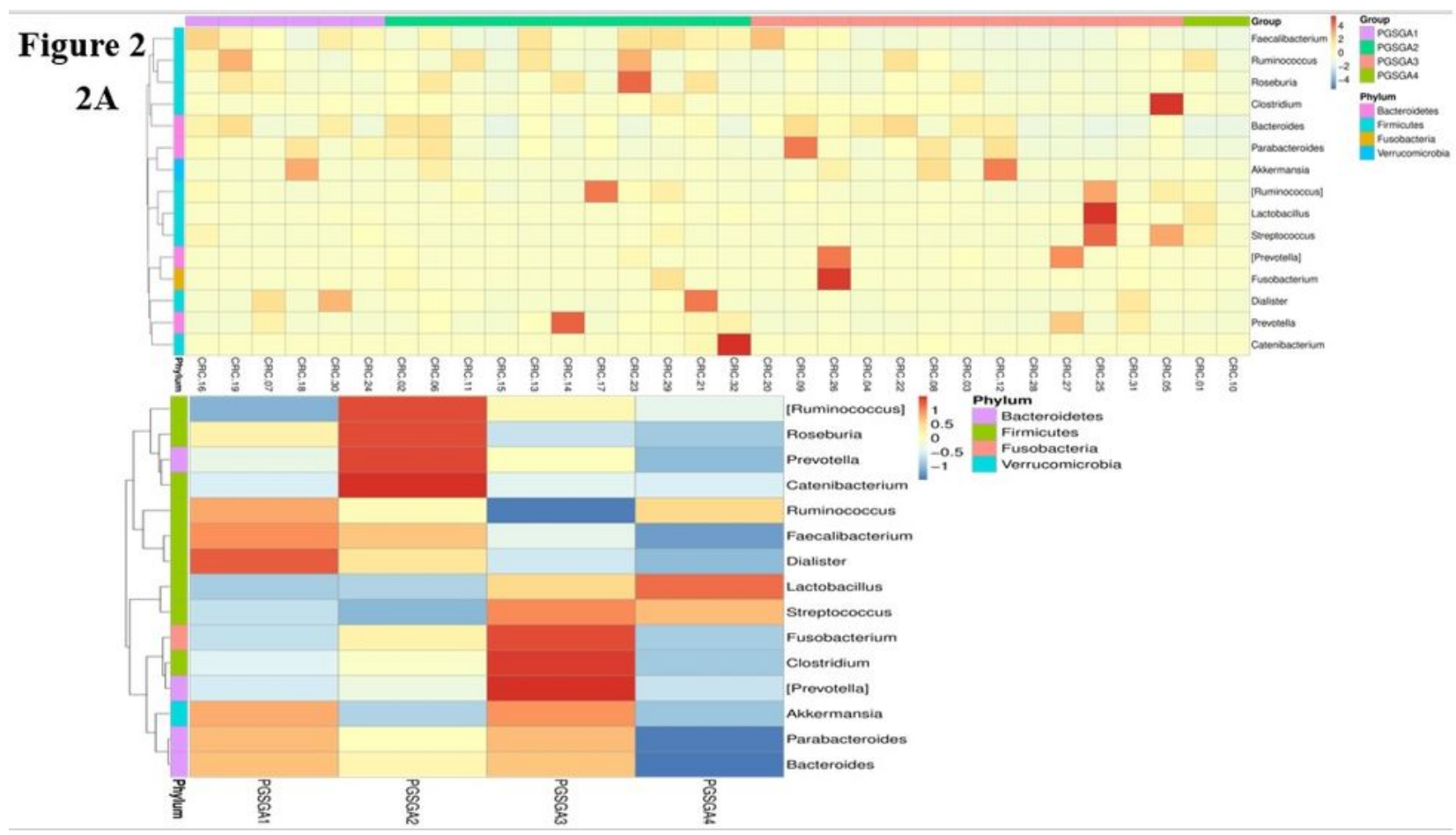

2B
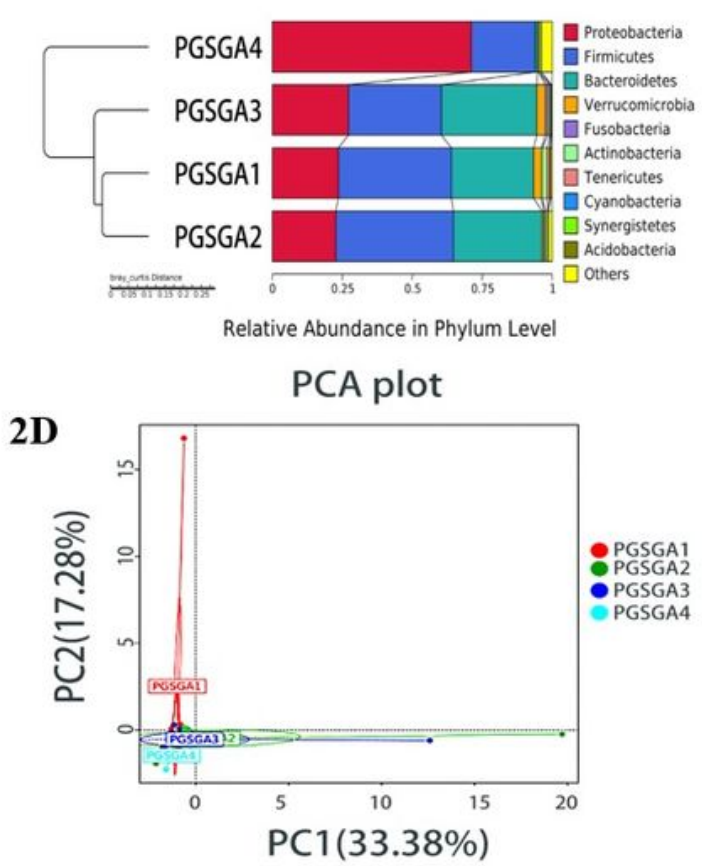

2C

PCoA plot

$2 \mathbf{E}$
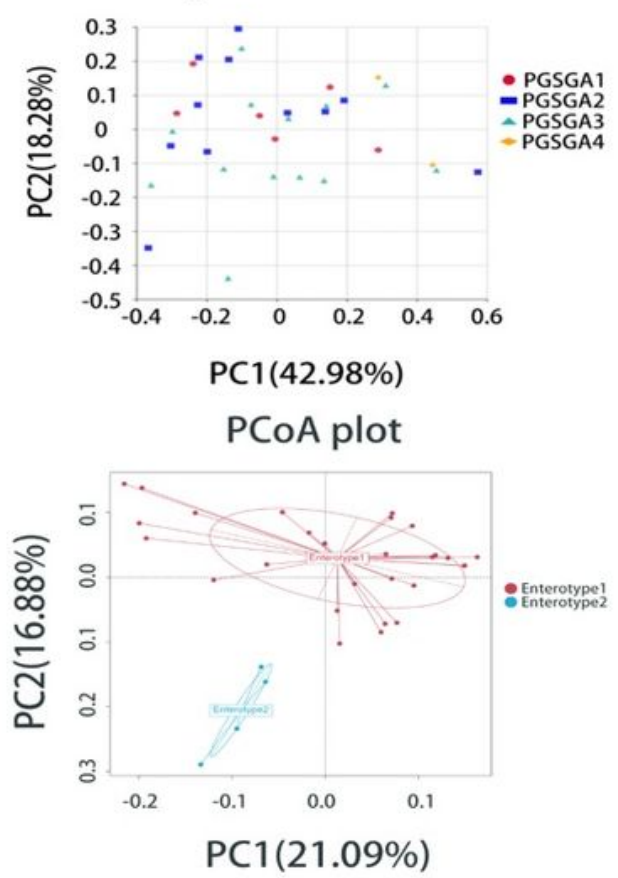

\section{Figure 2}

Comparison of fecal microbiota among the four groups assessed by PG-SGA screening scores. A: Heatmap of fecal microbiota from all the CRC patients and four groups assessed by PG-SGA screening tool in genera levels. B: Bray Curtis analysis of four groups assessed by PG-SGA. C: Principal Coordinates Analysis scores based on the relative abundance of the fecal microbiota at the genera levels. D: 
Principal Component Analysis scores based on the relative abundance of the fecal microbiota at genera levels. E: Gut microbiotas were calculated and divided into two groups (enterotype).

Figure 3

3A

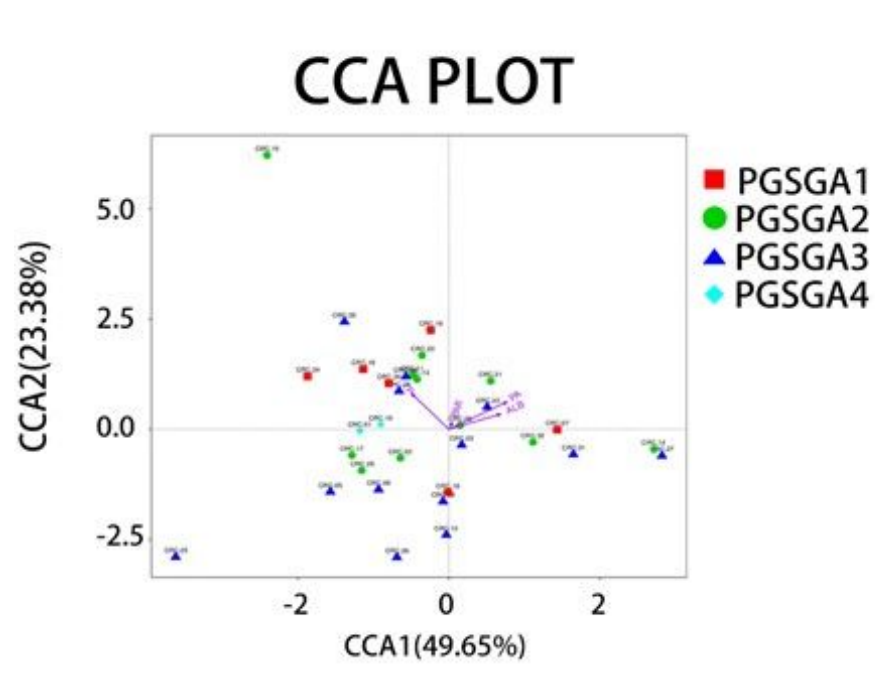

3B

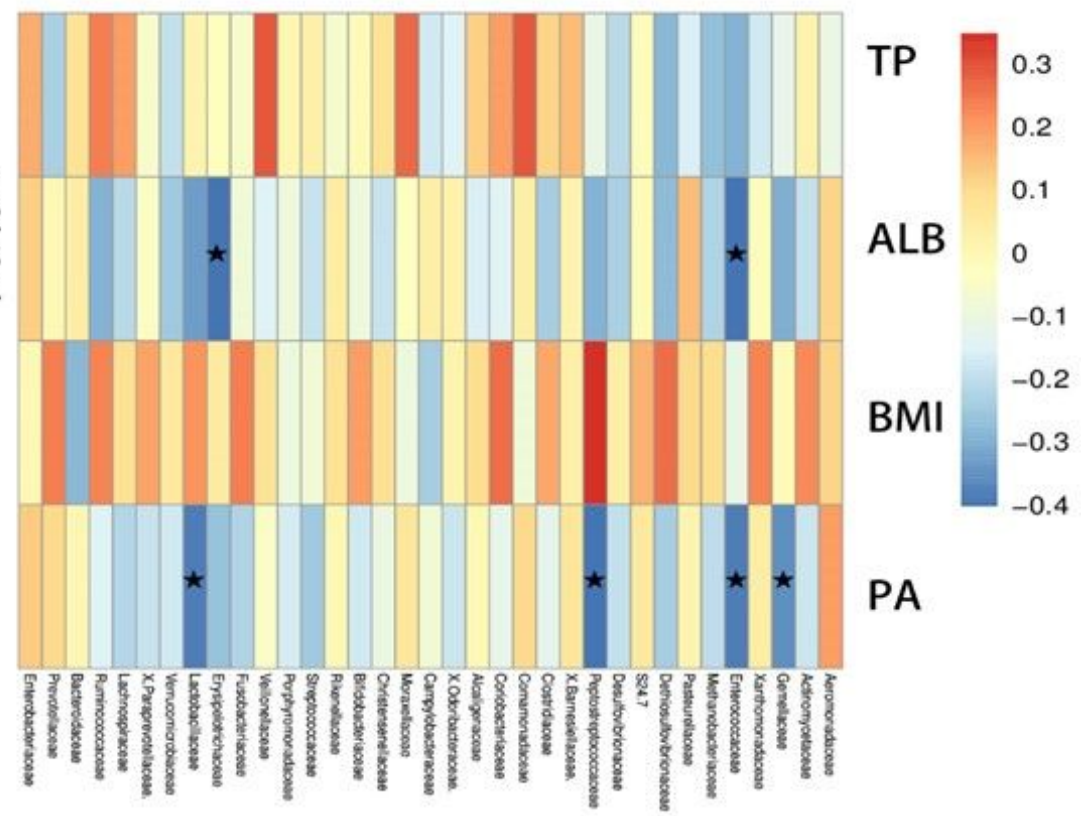

\section{Figure 3}

Correlation of clinical nutrition indicator with microbes. A: Canonical Correspondence Analysis between the two groups assessed by PG-SGA screening scores. B: Pearson correlation analysis was performed to assess the correlation of clinical nutrition indicator with microbes in all samples $(*, P<0.05)$. 


\section{Figure 4}

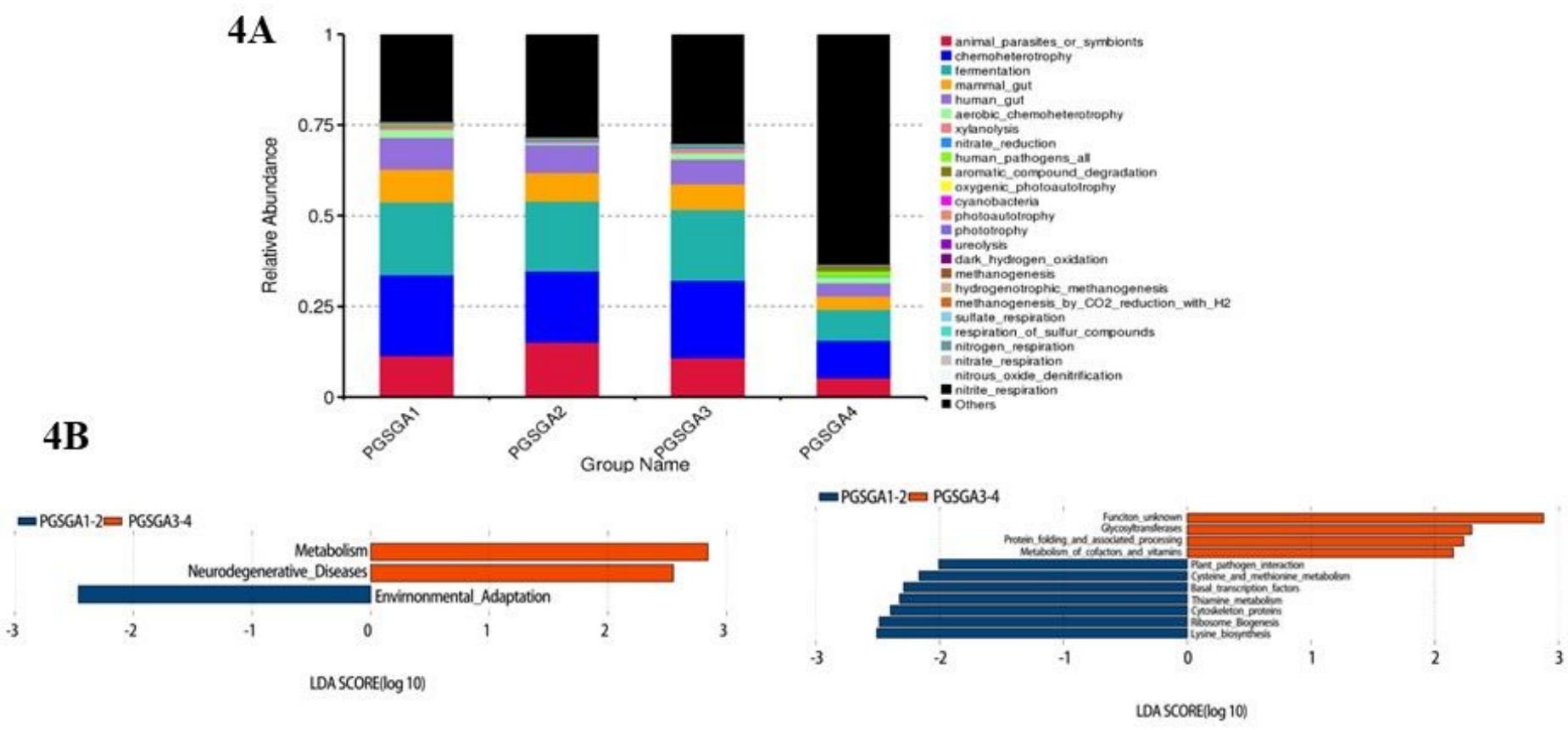

\section{Figure 4}

Metabolism analysis of the microbe in four groups assessed by PG-SGA. A: Correlation of metabolic signaling with the microbe in four groups assessed by PG-SGA. B: Linear discriminant Analysis of the microbe in different PG-SGA group. 
Figure 5

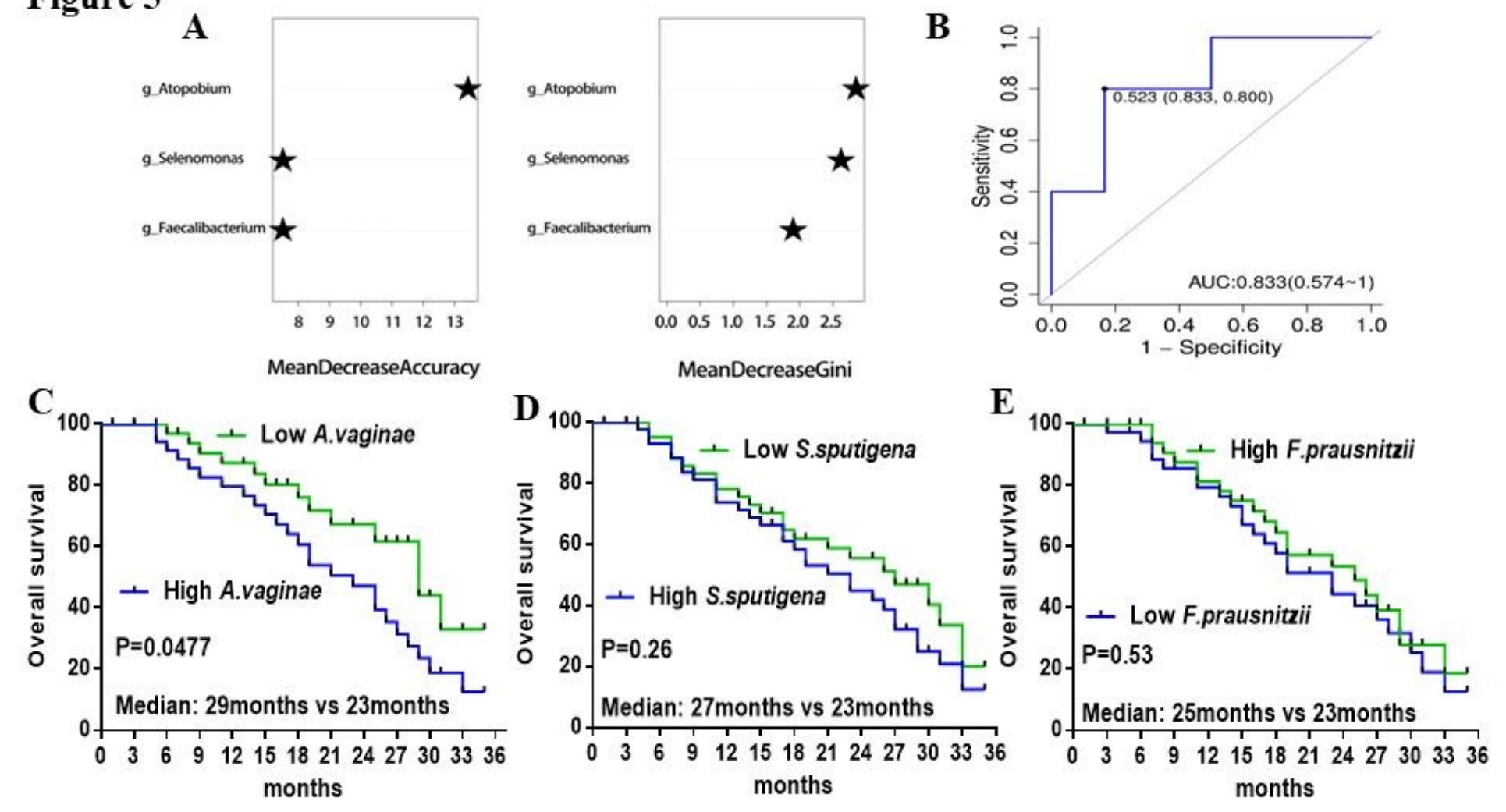

\section{Figure 5}

Establishing of disease surveillance model in malnutrition. A-B: Receptor operating characteristic curve (ROC curve) in prediction of different species in different groups assessed by PG-SGA. C-E: kaplan-meier curve to analyze the selected three microbiota such as Atopobium (C), Selenomonas (D) and Faecalibacterium (E) in CRC patients. 


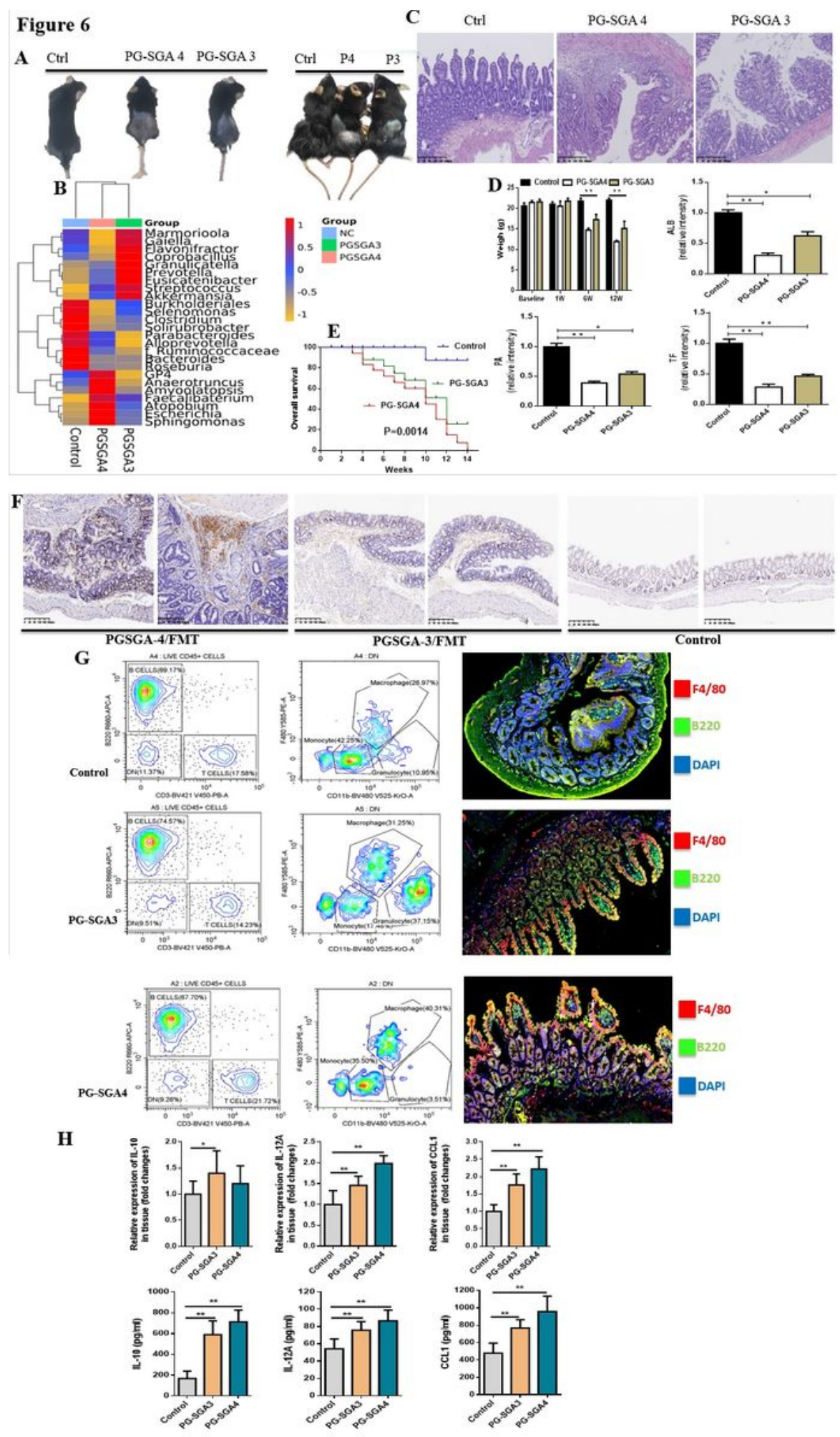

\section{Figure 6}

Gut microbiota from malnutrition considered as the co-carcinogenic factor. A: The autoimmune response of C57BL/6J mouse after FMT with the fecal from CRC patients assessed as PG-SGA4 or PG-SGA3. B: $16 \mathrm{~S}$ rRNA sequencing of C57BL/6J mouse after FMT with the fecal from CRC patients assessed as PGSGA4 or PG-SGA3. C: Pathological change examined by HE stain of C57BL/6J mouse after FMT with the fecal from CRC patients assessed as PG-SGA4 or PG-SGA3. D: The change of nutritional indicator of 
C57BL/6J mouse after FMT with the fecal from CRC patients assessed as PG-SGA4 or PG-SGA3. E: The overall survival analysis of $\mathrm{C} 57 \mathrm{BL} / 6 \mathrm{~J}$ mouse after FMT with the fecal from CRC patients assessed as PGSGA4 or PG-SGA3. F: Ki-67 expression of each tissue from each group examined by IHC. G: Accumulation of immune cells in serum from each group examined by FACS and tissues tested by immunofluorescence. H: Expression of cytokines in serum from each group examined by ELISA and tissues tested by qPCR. $\left({ }^{*}, \mathrm{P}<0.05, * *, \mathrm{P}<0.01\right)$.
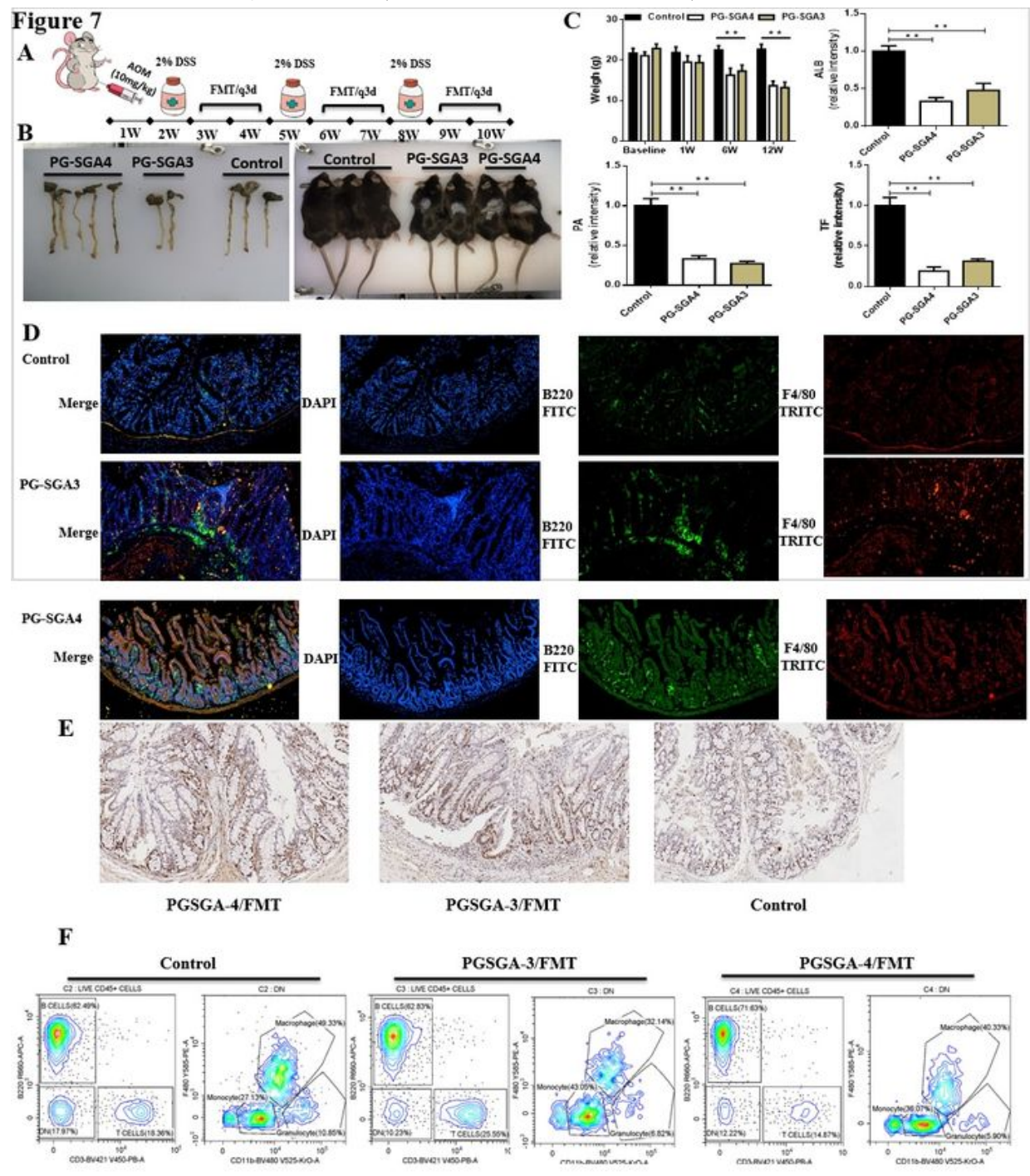

G

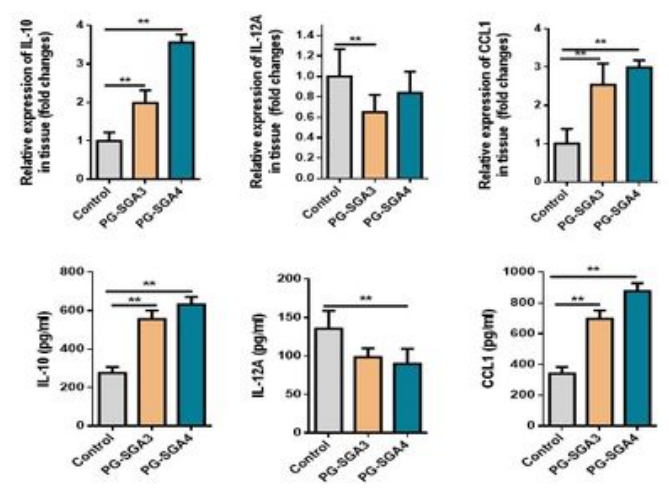

Figure 7 
Gut microbiota from malnutrition accelerating the CRC progression in vivo. A: The constructive project of DSS/AOM mice models as well as the FMT schedule. B: The amount of adenoma and autoimmune response of DSS/AOM mice models after FMT with the fecal from CRC patients assessed as PG-SGA4 or PG-SGA3. C: The change of nutritional indicator of DSS/AOM mice models after FMT with the fecal from CRC patients assessed as PG-SGA4 or PG-SGA3. D: Accumulation of immune cells in tissues tested by immunofluorescence. E: Ki-67 expression of each tissue from each group examined by IHC. F: Accumulation of immune cells in serum from each group examined by FACS. G: Expression of cytokines in serum from each group examined by ELISA and tissues tested by qPCR.

\section{Figure 8 PBS}
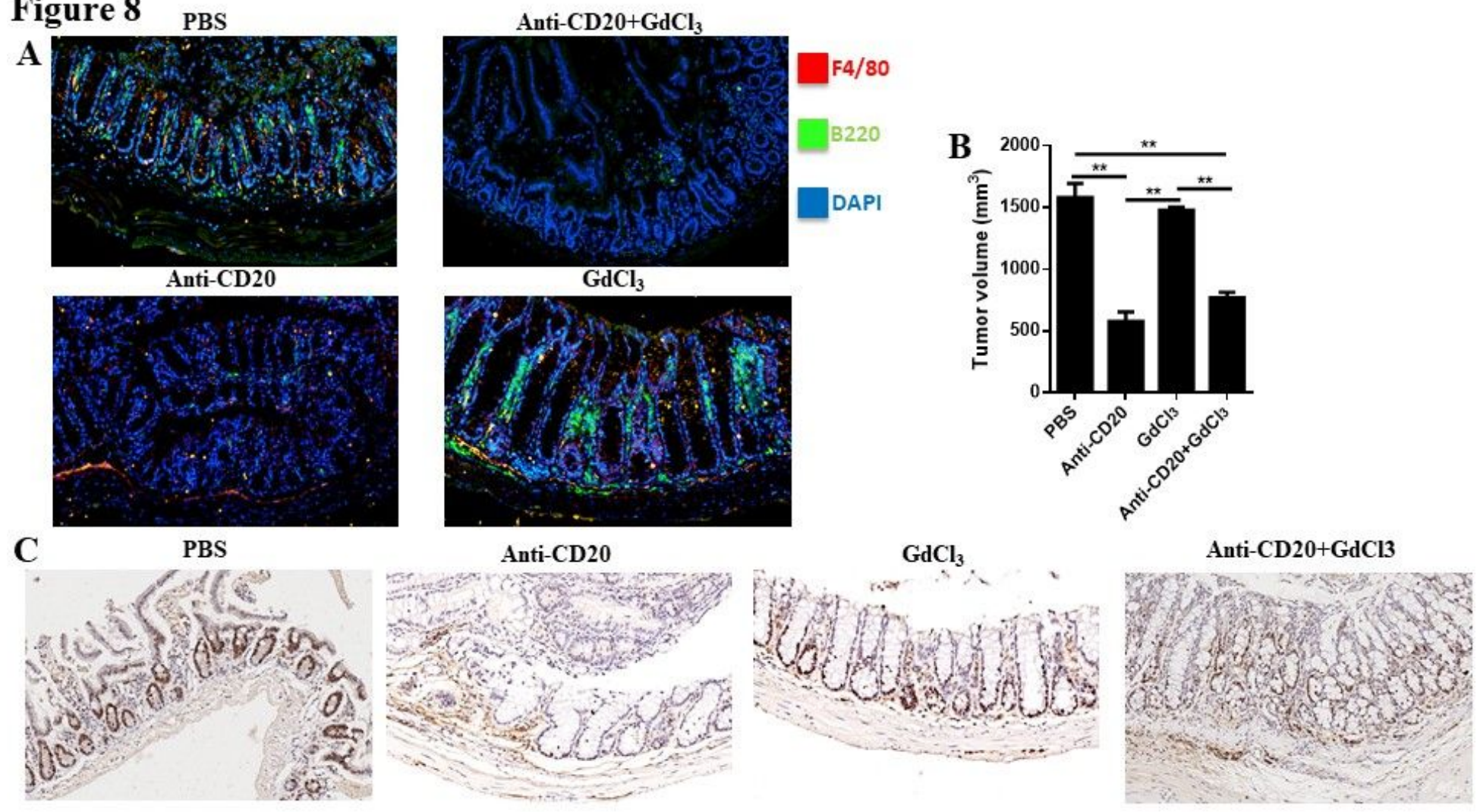

Figure 8

Suppression of B cells or inhibition of monocyte/macrophage activities attenuating the CRC progression induced by microbiota. A: Accumulation of immune cells in tissues tested by immunofluorescence after PG-SGA4 FMT with treatment of anti-CD20 or GdCl3 or anti-CD20 plus $\mathrm{GdCl} 3$. B: The tumor volume changes after PG-SGA4 FMT with treatment of anti-CD20 or GdCl3 or anti-CD20 plus GdCl3. C: Ki-67 expression after PG-SGA4 FMT with treatment of anti-CD20 or GdCl3 or anti-CD20 plus GdCl3.

\section{Supplementary Files}

This is a list of supplementary files associated with this preprint. Click to download.

- AppendixTables1.docx

- AppendixTables1.docx 